\title{
(Re)organizando a sala de curativo do Centro de Saúde Escola da Faculdade de Medicina de Ribeirão Preto da Universidade de São Paulo
}

\author{
Ione Carvalho Pinto ${ }^{1}$ \\ Ivana Astolphi Gandra Passeri² \\ Daniela Soares da Silva ${ }^{3}$ \\ Michele Mandagará de Oliveira ${ }^{4}$ \\ Pinto IC, Passeri IAG, Silva DS, Oliveira MM. (Re)organizando a sala de curativo do Centro \\ de Saúde Escola da Faculdade de Medicina de Ribeirão Preto da Universidade de São Paulo. \\ Acta Paul Enferm 2005; 18(1):89-93.
}

\begin{abstract}
RESUMO:O presente estudo é um relato de experiência da reorganização da assistência de enfermagem na sala de curativos do Centro de Saúde Escola da Faculdade de Medicina de Ribeirão Preto da Universidade de São Paulo. Teve como objetivo geral implantar a sistematização da assistência de enfermagem, segundo o protocolo da Secretaria Municipal de Saúde do município de Ribeirão Preto do Estado de São Paulo. Identificamos a demanda de acordo com os aspectos bio-psico-social e classificação das feridas. Realizamos seleção (teórico-prática) entre os funcionários e o levantamento de recursos físicos e materiais necessários para organização da sala, assim como a formação de equipe multiprofissional para suporte técnico ao cuidado de enfermagem na sala de curativo. (Re) organizar a sala de curativos passa não somente pelo compromisso de implantar medidas de intervenção local e meramente técnicas, mas também por intervenções macro-estruturais do sistema de saúde,buscando, através dos princípios do Sistema Único de Saúde, reorganizar a assistência, objetivando traçar estratégias e metas para melhorar a saúde da população.
\end{abstract}

Descritores: Cuidados de enfermagem; Serviços de enfermagem; Organização e administração

- Artigo recebido em 21/02/03 e aprovado em 26/06/03

\section{INTRODUÇÃO}

Ao iniciarmos o Serviço de Coordenação de Enfermagem do Centro de Saúde Escola da Faculdade de Medicina de Ribeirão Preto da Universidade de São Paulo (CSE-FMRPUSP), uma docente da Escola de Enfermagem de Ribeirão Preto da Universidade de São Paulo (EERP) e uma enfermeira contratada pela mesma escola para coordenação, em tempo integral, da equipe de enfermagem, identificamos a necessidade de (re) organizar a assistência de enfermagem prestada ao portador de feridas na sala de curativos, proporcionando um cuidado humanizado e sistematizado norteado pelos princípios do Sistema Único de Saúde, tendo como base de sustentação técnica o protocolo de curativo da Secretaria Municipal de Saúde de Ribeirão Preto-SP (SMS-RP-SP).

A organização dos serviços de saúde compreende um conjunto de ações desenvolvidas com o objetivo de permitir a estruturação física e o desenvolvimento dos trabalhadores, através de uma alocação adequada dos recursos necessários para a prestação da assistência à saúde da população, considerando este conjunto de ações como "ferramentas" para a construção de um atendimento mais humanizado ${ }^{(1)}$. Para isso, foi necessário buscar possibilidades para transformar a lógica do modelo hegemônico anterior, onde apenas a queixa pontual do usuário, sua ferida, era avaliada e tratada.

\footnotetext{
1 Professora Doutora do Departamento de Enfermagem Materno-Infantil e Saúde Pública da Escola de Enfermagem de Ribeirão Preto da Universidade de São Paulo e Coordenadora de Enfermagem do Centro de Saúde Escola-FMRP-USP.

Enfermeira Coordenadora de Enfermagem do Centro de Saúde Escola-FMRP-USP.

3 Enfermeira Responsável pela Sala de Curativo do Centro de Saúde Escola-FMRP-USP.

4 Enfermeira Mestranda do Departamento de Enfermagem Materno-Infantil e Saúde Pública da Escola de Enfermagem de Ribeirão Preto da Universidade de São Paulo. Bolsista do CNPq.
} 
Desta forma, foram realizadas reuniões com todos enfermeiros do CSE-FMRP-USP, uma vez que o envolvimento deste grupo seria fundamental para viabilização das transformações, e também para auxílio em algumas tarefas como, por exemplo, levantamento do número dos usuários da sala de curativo, supervisão na seleção (teórico-prática) para escolha de auxiliares ou técnicos de enfermagem responsáveis pelo curativo e participação na equipe multidisciplinar formada e organizada para oferecer suporte aos técnicos diretamente envolvidos com o cuidado.

A complexidade dos problemas de saúde exige, no seu enfrentamento, uma abordagem diferenciada que permita reconstruir o conhecimento da determinação social do processo saúde-doença e suas conexões, incorporando visões interdisciplinares e apoio intersetorial. Este processo irá se

refletir em ações setoriais e intersetoriais porque freqüentemente, um problema de saúde vai exigir ações articuladas que estão situadas fora dos limites convencionais do setor ${ }^{(2)}$.

Assim sendo, tornou-se imprescindível formar uma equipe de trabalho com visão interdisciplinar (Médico Dermatologista, Enfermeiro, Auxiliar de Enfermagem, Assistente Social e outros profissionais de saúde), apoiada pela Escola de Enfermagem de Ribeirão Preto da Universidade de São Paulo (Docentes e alunos de graduação e de pós-graduação do Departamento de Enfermagem Materno-Infantil e Saúde Pública e do Departamento de Enfermagem Especializada e Geral) e, sempre que necessário, a equipe contou, também, com o apoio de outras especialidades e serviços (Clínica Médica, Vascular, Psiquiatria, Psicologia, Nutrição e Grupos Educativos).

\section{OBJETIVOS DA \\ (RE) ORGANIZAÇÃO \\ DA SALADE CURATIVO}

- Sistematizar a assistência de enfermagem segundo o protocolo da SMS-RP-SP

- Acolher o usuário, estabelecer vínculo, garantir seguimento

- Otimizar recursos humanos

- Supervisionar a técnica do curativo, capacitar o profissional que atua na sala de curativo

- (Re) ordenar a demanda

- Favorecer o ensino acadêmico

\section{APRESENTAÇÃO DO CENTRO DE SAÚDE ESCOLA DA \\ FACULDADE DE MEDICINADE RIBEIRÃO PRETO DA UNIVERSIDADE DE SÃO PAULO}

Desde sua inauguração em 1979, o CSE-FMRP-USP atua como Distri-tal de Saúde, oferecendo assistência à saúde da população do distrito oeste do município de Ribeirão Preto-SP. Sua área distrital conta com uma população estimada em 130.688 habitantes.

Sua área básica abrange uma população de aproximadamente 18.006 habitantes, distribuídos em oito bairros, compreendendo 11 Unidades Básicas de Saúde e 4 Núcleos de Saúde da Família. Oferece um serviço de pronto atendimento que funciona diariamente durante 24 horas, e um serviço de área programática que oferece agendamentos para as seguintes especialidades médicas: oftalmologia, cardiologia, dermatologia, ginecologia, pediatria, ortopedia.

O CSE-FMRP-USP, ainda oferece serviços de enfermagem relacionados às salas de vacina, medicação e observação, coleta de exames laboratoriais e de curativos assépticos e sépticos, na qual se dá, atualmente, a realização de curativos padronizados pela SMS-RP-SP. Estamos relatando, no presente artigo, toda a reorganização para o funcionamento da sala de curativos.

\section{PASSOS DA(RE)OGANIZAÇÃO \\ DA ASSISTÊNCIA DE ENFERMAGEM}

1. Reuniões semanais com os enfermeiros durante os meses de setembro, outubro, novembro, e dezembro de 2001.

2. Levantamento de dados sobre as condições de saúde, econômicas e sociais dos usuários, a partir dos prontuários de atendimento, assim como endereço dos seus domicílios e verificação se os mesmos pertenciam à área básica de cobertura do CSE-FMRP-USP.

3. Reorganização da estrutura física da sala de curativos, como limpeza, desinfecção, e reorganização dos materiais permanentes da sala.

4.Controle de recursos materiais para reorganizar e oferecer a assistência de qualidade ao portador de feridas crônicas.

5.Escolha das datas para realização da avaliação teórico-prática para escolha do profissional técnico ou auxiliar de enfermagem responsável pelo cuidado direto ao portador de feridas crônicas.

6.Preparação das avaliações teóricopráticas de acordo com o protocolo de curativo da SMS-RP-SP.

7.Capacitação teórica por meio de cursos e treinamentos em serviço oferecidos, tanto pela SMS-RP-SP, quanto pelas próprias enfermeiras do CSE-FMRP-USP.

8.Formação de equipe multiprofissional de trabalho. Para esta formação, foram convidados além de alguns profissionais do próprio CSEFMRP-USP (Médico dermatologista, três Enfermeiras, uma Auxiliar de enfermagem), docentes (Enfermeira e Nutricionista) e alunos do mestrado do Departamento de Enfermagem Materno Infantil e Saúde Pública, assim como alunos do doutorado do Departamento de Enfermagem Geral e Especializada e alunos de graduação da EERP-USP. 
Também fazem parte do grupo uma Enfermeira do nível central da Secretaria Municipal da Saúde de Ri- beirão Preto e uma Enfermeira do Núcleo de Saúde Mental da região oeste de Ribeirão Preto. Reuniões mensais foram realizadas com a equipe multiprofissional de apoio, desde agosto de 2002.

\section{A OPERACIONALIZAÇÃO DO FLUXO DAS AÇÕES - DESDE A PORTA DE ENTRADA DO SERVIÇO ATÉ AALTA OU TRANSFERENCIA DO USUÁRIO PARA OUTRO NÍVEL DE ASSISTÊNCIA}

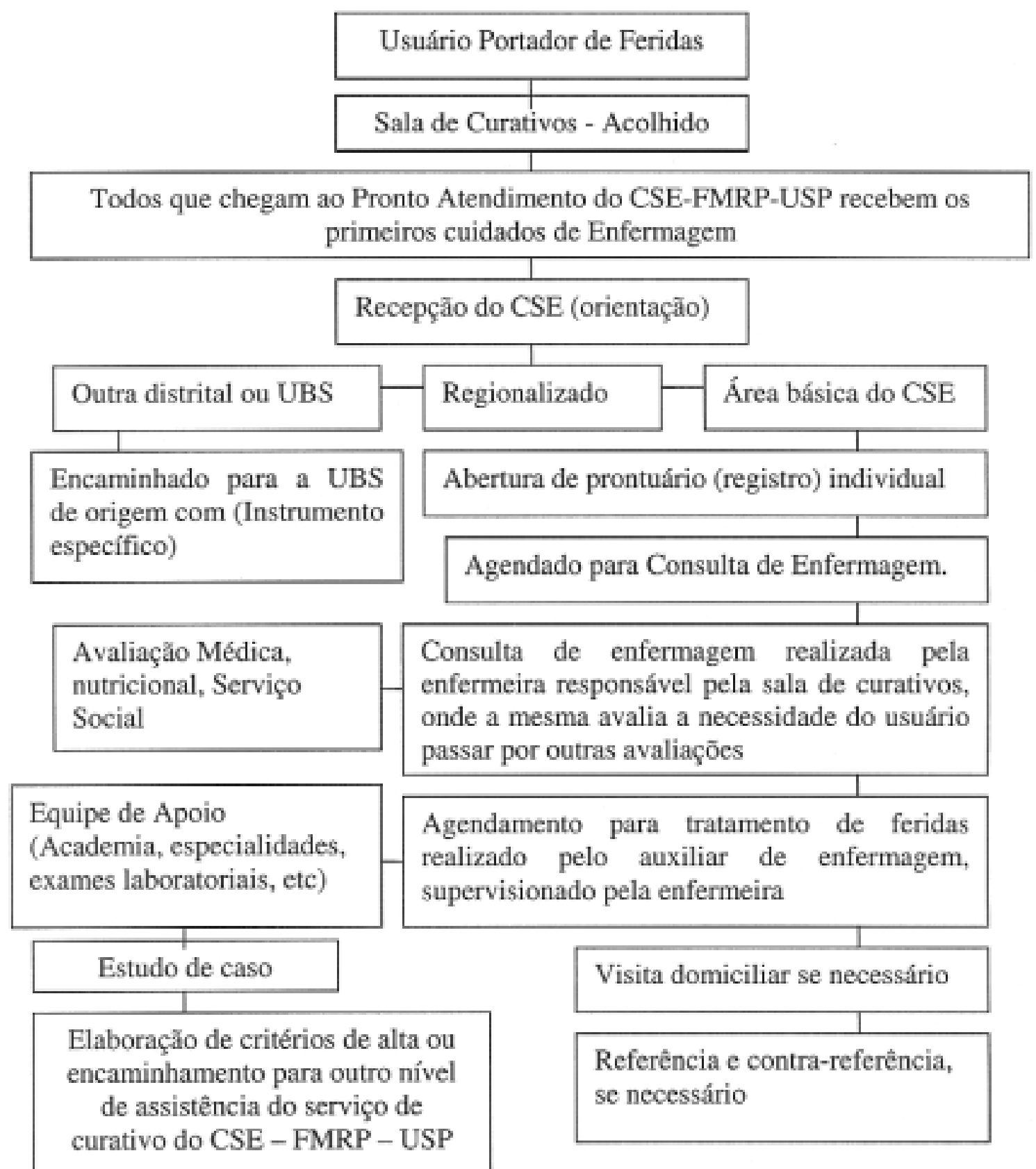

Figura 1 - Fluxograma da assistência aos pacientes portadores de feridas no Centro de Saúde Escola da FMRP-USP 


\section{MONITORAMENTO DO PROCESSO DE (RE) ORGANIZAÇÃO DA SALADE CURATIVOS}

A sistematização da assistência na sala de curativo foi iniciada em abril de 2002. Em janeiro de 2003 estávamos atendendo 27 usuários, todos portadores de feridas crônicas (17 apresentavam úlcera venosa, 06 feridas traumáticas, 03 úlcera neuropática e um apresentava úlcera de pressão), residentes na área distrital. $\mathrm{O}$ atendimento era realizado pela técnica de enfermagem e enfermeira responsável pelo setor, com supervisão da coordenação de enfermagem. Os casos novos foram consultados e avaliados pela enfermeira através da consulta de enfermagem, também previamente agendada. No seguimento dos usuários foram utilizados os seguintes instrumentos:

- Ficha de avaliação e do perfil sócio-econômico do usuário;

- Ficha de anotações gerais: Visita domiciliar e outras;

- Guia de encaminhamento médico e de enfermagem;

- Carteira de agendamento e Carteira de vacina.

Todo usuário que procura o Serviço da sala de curativos do CSEFMRP-USP, primeiramente é acolhido pela técnica de enfermagem que, se necessário, presta cuidado imediato. Logo após, o mesmo é encaminhado à recepção do serviço para ser regionalizado à UBS de sua referência. $\mathrm{O}$ usuário que pertence à área básica é agendado no serviço para acompanhamento e tratamento da ferida.

\section{CONSIDERAÇÕES GERAIS}

(Re) organizar a sala de curativos do CSE-FMRP-USP, passa não somente pelo compromisso de implantar medidas de intervenção local e meramente técnicas, mas também por intervenções macro-estruturais do sistema de saúde através de fóruns, favorecendo abertura do diálogo entre os trabalhadores da saúde, comunidade e representantes da Secretaria Municipal Saúde, objetivando traçar estratégias e metas para melhorar a saúde da população.

Foi com esse intuito que, durante alguns encontros com os enfermeiros do serviço, conseguimos perceber, através de suas falas, que os mesmos estavam muito preocupados com a aderência dos usuários ao tratamento. Não havia um profissional fixo na sala, o que, muitas vezes, dificultava a melhora da ferida, mesmo que a técnica de curativo estivesse sendo realizada da maneira correta. Também não havia registro de informações gerais e específicas em fichas individuais, necessárias para continuidade e avaliação do cuidado aos portadores de feridas crônicas. O usuário era tratado de modo muito pontual, apenas sua queixa era tratada, não havia acompanhamento do caso, muitos dos usuários pertenciam a outras UBDS ou UBS e até mesmo eram residentes em outro município vizinho a Ribeirão Preto.

A oportunidade de repensar nossos serviços foi importante neste processo, considerando que muitos estão organizados a partir de problemas específicos que podem estar dentro da ótica hegemônica do modelo médico neoliberal, individualista, curativo, centrado apenas na doença ${ }^{(3)}$.

A dimensão da concepção saúde-doença é

a mais importante na estruturação dos Modelos Tecnoassistenciais e no que tange à capacidade resolutiva de problemas (eficácia), pois quanto maior sua capacidade explicativa de fenômenos que interferem no estado de saúde, maior sua capacidade de formular alternativas de solução ${ }^{(4)}$.
A possibilidade de reconstruir e reorganizar a assistência na sala de curativo com um cuidado mais humanizado, além de ser um projeto piloto de implantação de protocolo da Secretaria Municipal de Saúde de Ribeirão Preto-SP para o cuidado de feridas crônicas, também foi uma grande aposta da equipe dos profissionais do CSE-FMRP-USP e da equipe de apoio do grupo de curativos de que é possível transformarmos a lógica biologicista, em outra lógica, que consiga assistir o usuário na sua totalidade, no seu meio social, na sua particularidade.

Com isso, o grupo também pretende motivar os outros profissionais de que é possível transformar este modelo hegemônico dentro dos serviços de saúde. Para isso, temos que rever nossos conceitos de saúde-doença e (re)pensar como está sendo oferecida a assistência, para isso, é importante acreditarmos que a mudança é possível.

\section{REFERÊNCIAS}

1. Mishima SM. Contribuição do gerenciamento local na rede básica de saúde de Ribeirão Preto [tese doutorado]. Ribeirão Preto: Escola de Enfermagem de Ribeirão Preto/USP; 1995.

2. Mendes EV. Distritos sanitários: o processo social de mudança das práticas sanitárias do Sistema Único de Saúde. São Paulo: Hucitec-Abrasco; 1995.

3. Campos GWS. Reforma da reformarepensando a saúde. São Paulo: Hucitec; 1997.

4. Silva AG Junior. Modelos técnicos assistenciais em saúde-o debate no campo da saúde coletiva. São Paulo: Hucitec; 1998. 
Pinto IC, Passeri IAG, Silva DS, Oliveira MM.[(Re) organizing the wound dressing unit of the Health Care Center/University of São Paulo at Ribeirão Preto School of Medicine.] Acta Paul Enferm 2005; 18(1):89-93.

ABSTRACT: This study is a report of experience about the reorganize the nursing care provided at the wound dressing unit of the Health Care Center/University of São Paulo at Ribeirão Preto Schoo of Medicine. The general goal of this research was to implement the systematization of nursing care according to the protocol of the Municipal Health Center. Aiming at making the study viable, at first the demand was identified according to the bio-psycho-social aspects and the wound classification. Afterwards, authors selected (a theoretical and practical selection) among the workers, the physical and material resources that were necessary to organize the unit as well as to form a multiprofessional team to provide technical support to the nursing care. Therefore, reorganizing the wound dressing unit involves not only the commitment to implement measures of local and technical interventions but also macrostructural interventions in the health system, finding through the principles of the Unified Health System the elements to reorganize the care, aiming at developing strategies and improving the population's health.

Descriptors: Nursing care; Nursing services; Organization and administration
Pinto IC, Passeri IAG, Silva DS, Oliveira MM. [(Re) organización de la sala de curativo del Centro de Salud Escuela de la Facultad de Medicina de Ribeirão Preto de la Universidad de São Paulo.] Acta Paul Enferm 2005; 18(1):89-93.

RESUMEN: El presente estudio es un informe de experiencia sobre la reorganización de lo cuidado de enfermería en la sala de curativos del CSE-FMRP-USP y tuvo como objetivo general implantar la sistematización de la asistencia de enfermería según el protocolo de la SMS - RP. Para viabilización de este estudio primeramente la demanda fue identificada de acuerdo con los aspectos bio-psico-sociales y clasificación de las heridas, a seguir fue realizada la selección (teórico-práctica) entre los funcionarios, levantamiento de recursos físicos y materiales necesários para organización de la sala, así como formación de equipo multiprofesional para soporte técnico a asistencia de enfermería de la sala de curativo. Así siendo, (re) organizar la sala de curativos involucra, además del compromiso de implantar medidas de intervención local y meramente técnicas, las intervenciones macro-estruturales del sistema de salud, buscando a través de los princípios del SUS reorganizar la asistencia, objetivando delinear estratégias y metas para mejorar la salud de la población.

Descriptores: Atención de enfermería; Organización y Administración; Servicios de enfermería 